\title{
Preface
}

\section{The MERSEA Project}

\author{
Y. Desaubies \\ Project Manager, IFREMER, France
}

\section{Introduction}

The MERSEA project (2004-2008) was funded by the European Commission sixth Framework Programme (FP6) in response to its Aeronautics and Space Call for the development of Ocean and Marine Applications for GMES (Global Monitoring for Environment and Security). A consortium of forty Agencies and Laboratories from sixteen countries was formed to elaborate and carry out an ambitious work plan structured along twelve work packages.

The terms of the Call placed strong emphasis on the development of applications and services.

Accordingly, the strategic objective of MERSEA was stated as "to provide an integrated service of global and regional ocean monitoring and forecasting to intermediate users and policy makers in support of safe and efficient offshore activities, environmental management, security, and sustainable use of marine resources".

We aimed "to develop a European system for operational monitoring and forecasting on global and regional scales of the ocean physics, biogeochemistry and ecosystems. The prediction time scales of interest extend from days to months. This integrated system will be the Ocean component of the future GMES system".

During the elaboration of the MERSEA work plan we decided that several lines of action were required to reach those objectives by 2008 , including

- the provision of input data (from satellites, in situ and atmospheric forcing fields);

- research and development activities to achieve high quality and efficiency of all components and products;

- design, implementation and operation of the systems;

- development and demonstration of user oriented products.

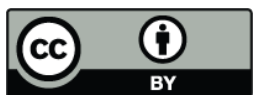

Correspondence to: Y. Desaubies (merseaconseil@orange.fr)
The development of ocean monitoring and forecasting systems on global and European regional scales calls for a broad range of research and development activities to ensure that they operate on firm scientific and technical grounds; that optimal use is made of all data available; that the systems are fully validated and robust from an operational standpoint; that they are well integrated into an efficient system of systems, with easy access and smooth exchange of data; and that the systems are fit for purpose with engagement of the stakeholders.

The conception of the project was based on the view that a prerequisite to the development of ocean and marine applications (as stated for in the Call for Proposal) is the provision of reliable, generic data and information products serving the needs of several classes of intermediate users, to enable them to fulfil their mission and to provide the services required by their final users.

\section{Data}

Ocean data come from three broad classes of sources: in situ platforms (buoys, ships, floats); satellites; and numerical weather prediction (NWP) from meteorological services. Those observations are valuable as unique global data sets, and are used as input for assimilation or forcing fields of predictive numerical ocean models.

For remote sensed data, the focus of the project has been on improving the retrieval algorithms required to determine with high accuracy the geophysical parameters (e.g. ice concentration and extent, ice drift, chlorophyll, suspended matter, sea surface temperature, sea surface height, mean dynamic topography). Whenever possible, data from different satellites and sensors are used to obtain uniform merged data products, mapped onto geographic grids.

The project could not support a large contribution to in situ observing networks, but a few operations were conducted. A set of Argo floats were deployed, most significantly in high latitudes, where a specific ice-detection algorithm was developed to allow for the first time data collection under the ice.

Published by Copernicus Publications on behalf of the European Geosciences Union. 
Updated climatology of the Atlantic and the Global oceans have been obtained by retrospective synthesis of the global Argo array data, revealing large scale patterns of variability.

As a European contribution to the Ocean Sites programme, three moored stations were maintained in representative locations in the North Atlantic, and two in the Mediterranean. The stations allow real time transmission of multiparameters, including bio-geochemical ones.

Several tests and operations at sea of gliders have been conducted, including several runs over $1000 \mathrm{~km}$ long in the Atlantic and the Mediterranean, where multi instruments operations were conducted.

Considering the cost of data collection at sea it is necessary to ensure that all global data are available easily to users in the shortest delays. In performing that task, the Coriolis in situ data centre has very significantly increased (by a factor of three) the amount of quality controlled data available in real time, a large part of that increase being related to the ramping up of the Argo array.

\section{System design, development, implementation and operation}

One of the main challenges of the project was to integrate into a coherent system of systems the various centres that were operating in different contexts and stages of development. The design has led to the final structure of a distributed system comprising Monitoring and Forecasting Centres (MFC) and Thematic Assembly Centres (TAC). The MFCs cover the global ocean and the main European seas (Arctic, North East Atlantic, Baltic, and Mediterranean); the TACs process the data from satellite remote sensing (sea-ice, ocean colour, altimetry, and sea surface temperature), and from global in situ networks. All the Centres fulfil common functions (Production and delivery, system management, monitoring, service provision, user desk, quality assessment). Common data formats have been agreed upon, and consistent documentation is available on the systems specification, their catalogues and inventories. The protocols for data exchange have been defined as the MERSEA Information Management system. Thus different classes of users can be served according to their needs, the primary function of the system being to deliver common baseline products and data to intermediate users, who can in turn develop bespoke services to final users.

The monitoring and forecasting centres have been upgraded in several respects: model resolution, assimilation of satellite and in situ data, more frequent analysis and forecasts, adoption of new modelling framework (NEMO). The improved performance has been achieved by the introduction of new parameterisation and algorithms resulting in higher efficiency and realism of the models (e.g. bottom and interior mixing, ice modelling, topographic effects, mixed layer dynamics, advection schemes, assimilation techniques).
All systems include bio-geochemical modelling, some in a demonstration mode, since those models still require extensive validation. Nonetheless, the primary ecosystem forecasts have been introduced in the operational suite at the UK Met Office for the Northwest shelves, and in a preoperational mode at the Italian national Institute for Geophysics and Volcanology (INGV) for the Mediterranean.

The systems evolution and performance has been regularly evaluated for quality and consistency, with the aid of metrics, a methodology which has been adopted by the Global Ocean Data Assimilation Experiment (GODAE).

At the end of the project, all system components are operating continuously, delivering high quality data, analysis and forecasts over the global ocean and regional seas.

\section{Research on ocean modelling and data assimilation}

While research has been conducted regularly in all work packages, most notably to develop high quality data sets from remote sensing observations, specific activities have been carried out in the domains of ocean modelling, including bio-geochemical, data assimilation, and seasonal forecasting. Some of the results have been directly transferred to the operational suites, leading to more accurate representation of processes, and more efficient computing. However it is recognized that research operates on longer time scales than implementation and production; some of the developments will bear fruits in future versions of the systems. Promising results have been obtained in ecosystem modelling (class size approach), in advanced data assimilation schemes, in nesting and grid refinement, data assimilation in coastal models.

\section{Special Focus Experiments}

The Special Focus Experiments were devoted to the development of the coupling between the model system and the basic and generic model products of MERSEA with marine biogeochemical models for ecosystem forecasting, at the level of primary producers biomass and for the short time scales; and global atmospheric models for seasonal forecasting.

\section{Serving user needs}

Two broad classes of users have been considered in the project: those in the public sector, responsible for environmental monitoring and reporting; and maritime operations.

Several products and indicators have been developed in the perspective to support reporting requirements by the European Environment Agency (EEA), the marine Conventions, and the European Marine Strategy Directive. 
Several applications in the maritime sector have been explored: ship routing, offshore industry support, and oil spill drift prediction. In all cases the positive impact of high resolution ocean products has been demonstrated, but very stringent requirements are placed by users on accuracy, which cannot always be met by state of the art products. They also expect specific products tailored to their applications, and appropriate delivery mechanisms. Further investment and reliance by the industry on the Marine Core Services (as the GMES Marine Applications have now become to be known) hinges on the establishment of a reliable perennial service.

It is a measure of the success of the MERSEA project that it has produced so many original scientific results, high quality data sets, and is delivering an integrated system recognized as one of the most mature of the GMES components.

Many features of the MERSEA project are described in the following 11 research papers in this MERSEA special issue of Ocean science.
Acknowledgements. The Mersea project has been partially funded by the European Commission, under the FP6 contract AIP3-CT2003-502885. It has been led by the Mersea Executive Committee (P. Bahurel, M. Bell, E. Buch, Y. Desaubies, J. Johannessen, P.-Y. Le Traon, G. Manzella, N. Pinardi, S. Pouliquen, R. Rayner, H. Roquet, U. Send, and J. Verron). 